\title{
Single Chamber Power Injector
}

National Cancer Institute

\section{Source}

National Cancer Institute. Single Chamber Power Injector. NCI Thesaurus. Code C110924.

A pump system with one enclosed reservoir, designed to deliver intravenous contrast agent. 